\title{
'Basket studies' will hold intricate data for cancer drug approvals
}

The notion of targeting specific cellular mutations to make malignancies disappear got a boost in April when the director of the US National Cancer Institute (NCI), Harold Varmus, unveiled plans for the so-called MATCH trial. Announced at the annual meeting of the American Association for Cancer Research in Washington, DC, the trial aims to match at least 1,000 individuals with a variety of cancer types with therapies that target the specific mutations found in their tumors. In a separate study described by Varmus, the NCI will also genotype 100 'exceptional responders': trial participants who show noticeable improvements after treatment with cancer drugs that didn't provide much benefit to others, in the hope of finding mutations that explain why the medicines worked.

Such studies are meant to be exploratory, but they may offer a glimpse of the future. "What wed like to do is try a therapy based on the specific genetics or molecular features of your tumor and see if that will work better than flying blind," says Levi Garraway, a cancer geneticist at the Dana-Farber Cancer Institute in Boston.

However, as excitement grows about studies organized around cancer mutations rather than cancer type, it remains unclear how they will fit into the regulatory approval pathway. "This is terra incognita," says José Baselga, physician-inchief of the Memorial Sloan-Kettering Cancer Center in New York.

Baselga is pioneering this new type of genotype-focused clinical trial design. Last year, he and his colleagues launched a study to examine the effect of Zelboraf (vemurafenib), a drug from California's Genentech, in 101 patients with cancer with a mutation called $B R A F$ V600E. BRAF V600E is relatively common in individuals with melanoma, for which the drug was approved in 2011, but also occurs less frequently in other types of cancer. The drug showed little efficacy in a phase 1 trial involving patients with $B R A F$ mutant colorectal cancer (J. Clin. Oncol. 28, 15 s, 2010), but promising clinical activity in a recently published study involving three people with metastatic papillary thyroid cancer (PTC) harboring the mutation (Thyroid, http://doi. org/mh9, 2013). Baselga and his colleagues hope to find out who else the drug could help. The trial is open to individuals with multiple myeloma and almost any type of solid tumor that contains a BRAF V600E mutation, save melanoma and PTC.

This kind of trial is known as a 'basket study', and the approach is particularly useful when the cancer type or the mutation is rare. According

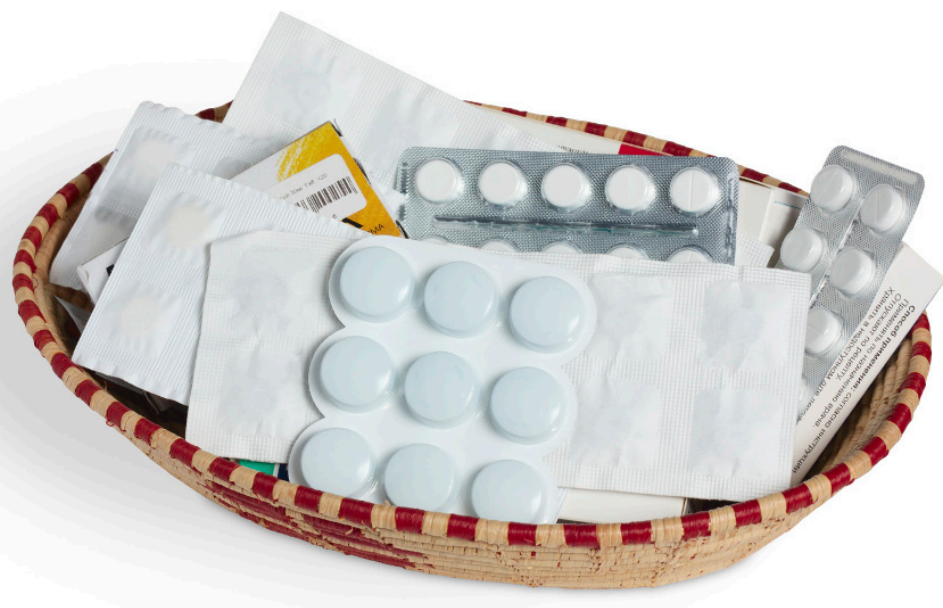

Basket case: Researchers weave a new trial design on the basis of genetics, rather than cancer type.

to Memorial Sloan-Kettering cancer researcher David Solit, the goal generally is to enroll about 10-15 subjects per tumor type, but such studies also typically include an 'other' category for patients with rare types of cancer in which the mutation of interest was not previously known to occur. In such cases, a randomized clinical trial-the gold standard for drug approvalsmay not be feasible because only a small number of people fit the profile for any given disease.

Regulatory agencies such as the US Food and Drug Administration (FDA) are not likely to approve a drug on the basis of data from only a couple of people. But if the drug worked for these participants, "then what you do is you enrich that cohort," Baselga says. In some cases, simply expanding the study to include more participants with a particular type of cancer might be sufficient for approval, especially when the results are striking and the need is great.

\section{A numbers game}

The FDA recognizes that recruiting study participants can be difficult in some cases. "We have approved drugs in small numbers of patients on single-arm trials cognizant of the fact that it would be difficult to enroll a large number of patients," says Richard Pazdur, head of the FDA's Office of Hematology and Oncology Products. In January 2012, for example, the FDA approved Genentech's Erivedge (vismodegib), the first drug for advanced basal cell carcinoma, off the back of positive efficacy results from a single-arm, phase 2 trial that had enrolled only 104 patients.

In other cases, the mutation or cancer type might be common enough to do a follow-up study. In 2010, Baselga launched a phase 1 basket study to examine the safety and efficacy of an experimental compound from Switzerland's Novartis called BYL719 in solid tumors that have mutations in the gene that encodes a subunit of the phosphatidylinositol 3-kinase protein. The drug seemed to work well in women with estrogen receptor-positive breast cancer, so Baselga and his colleagues now plan to test the efficacy of BYL719 combined with the hormone therapy fulvestrant in this subgroup.

It's not unfathomable that the FDA could approve a drug for a specific molecular target rather than a disease. "If we can show that what we do is safe and effective, I would suspect the FDA would welcome that," says Tomasz Beer, deputy director of the Oregon Health and Science University's Knight Cancer Institute in Portland. "It just has to be convincing."

Pazdur agrees. "There's nothing in the regulations that says a drug has to be approved in breast cancer or colon cancer," he says.

In 2006, the FDA approved Novartis's targeted tyrosine kinase inhibitor Gleevec (imatinib) for five types of cancer at once, including several rare malignancies. But whether any targeted medications will have broad enough activity to warrant more sweeping approval remains to be seen. As the case of Zelboraf in colorectal cancer shows, just because a single mutation occurs in more than one type of cancer doesn't necessarily mean that a medication that targets it will work in both. "It's not just the mutation that's important. The type of cancer you have is important also," says Solit.

Teasing apart the interactions between mutational profiles and tumor types is now a top priority for the cancer field. Like many things in medicine, conquering the biology is often more challenging than clearing the regulatory hurdles.

Cassandra Willyard 